\title{
dNTP pool modulation dynamics by SAMHD1 protein in monocyte-derived macrophages
}

Joseph A Hollenbaugh', Sijia Tao', Gina M Lenzi', Sulryung Ryư, Dong-Hyun Kim, Felipe Diaz-Griffero², Raymond F Schinazi ${ }^{1,3}$ and Baek Kim ${ }^{1,4^{*}}$

\begin{abstract}
Background: SAMHD1 degrades deoxyribonucleotides (dNTPs), suppressing viral DNA synthesis in macrophages. Recently, viral protein X (Vpx) of HIV-2/SIVsm was shown to target SAMHD1 for proteosomal degradation and led to elevation of dNTP levels, which in turn accelerated proviral DNA synthesis of lentiviruses in macrophages.

Results: We investigated both time-dependent and quantitative interplays between SAMHD1 level and dNTP concentrations during multiple exposures of Vpx in macrophages. The following were observed. First, SAMHD1 level was rapidly reduced by Vpx + VLP to undetectable levels by Western blot analysis. Recovery of SAMHD1 was very slow with less than 3\% of the normal macrophage level detected at day 6 post Vpx treatment and only $30 \%$ recovered at day 14 . Second, dGTP, dCTP and dTTP levels peaked at day 1 post Vpx treatment, whereas dATP peaked at day 2. However, all dNTPs rapidly decreased starting at day 3, while SAMHD1 level was below the level of detection. Third, when Vpx pretreated macrophages were re-exposed to a second Vpx treatment at day 7, we observed dNTP elevation that had faster kinetics than the first Vpx + VLP treatment. Moreover, we performed a short kinetic analysis of the second Vpx treatment to find that dATP and dGTP levels peaked at 8 hours post secondary VLP treatment. dGTP peak was consistently higher than the primary, whereas peak dATP concentration was basically equivalent to the first Vpx + VLP treatment. Lastly, HIV-1 replication kinetics were faster in macrophages treated after the secondary Vpx treatments when compared to the initial single Vpx treatment.
\end{abstract}

Conclusion: This study reveals that a very low level of SAMHD1 sufficiently modulates the normally low dNTP levels in macrophages and proposes potential diverse mechanisms of Vpx-mediated dNTP regulation in macrophages.

Keywords: HIV-1, Monocytes-derived macrophages, Vpx, SAMHD1, dNTPs: virus-like particles

\section{Background}

Sterile alpha motif (SAM) and histidine/aspartic acid (HD) domain protein 1 (SAMHD1) has been linked to Aicardi-Goutières Syndrome, which is a rare autoimmune disease [1]. In addition to its role in autoimmunity [2-4], SAMHD1 has been studied in the context of antiviral response [1,5-12] and genomic stability [2,13]. Several groups have now shown that SAMHD1 is found in all cell types and localizes to the nucleus [5,10,14-19].

\footnotetext{
* Correspondence: baek.kim@emory.edu

'Department of Pediatrics, Center for Drug Discovery, Emory Center for AIDS Research, Laboratory of Biochemical Pharmacology, Emory University School of Medicine, 1760 Haygood Drive, Health Sciences Research Building, Atlanta, Georgia 30322, USA

${ }^{4}$ College of Pharmacy, Kyung-Hee University, Seoul, South Korea

Full list of author information is available at the end of the article
}

Recent evidence indicates that SAMHD1 has at least two different cellular functions. First, SAMHD1 was shown to have deoxyribonucleoside triphosphate (dNTP) phosphohydrolase activity [20,21], suggesting it is a host antiviral restriction factor to limit replication of retroviral and DNA containing viruses by depleting cellular dNTPs in viral non-dividing target cell types [22-25]. Recently, both biochemical and structural evidence indicated that SAMHD1 forms a tetramer as the active dNTP phosphohydrolase complex [26-29]. When dNTPs bind in the active site, the tetramer is formed, and the tetramer was suggested to be long-lived in the cell [30]. SAMHD1 tetramer could maintain the cellular dNTP concentrations at a very low level outside the $\mathrm{S}$ phase of the cell cycle. Second, SAMHD1 was shown to have nuclease activity. The nuclease activity was localized to the HD domain of SAMHD1 [31]. Both single-stranded DNA and RNA 
nuclease activities have been reported for SAMHD1 $[29,32]$.

Regulation of SAMHD1 occurs by three mechanisms. First, promoter methylation was shown to inhibit transcription [33], leading to a reduction in SMAHD1 levels. Second, SAMHD1 is regulated during S phase [34,35], being targeted for degradation. Third, SAMHD1 phosphorylation at T592 [35] was shown to regulate its antiviral activity but not dNTP phosphohydrolase activity [11]. Importantly, White et al. have shown non-dividing cells do not phosphorylate SAMHD1 at T592, whereas cycling cells do [11].

HIV-2 and some SIV strains encode for the accessory viral protein $\mathrm{X}(\mathrm{Vpx})$. It has the ability to target human SAMHD1 to the proteasome for degradation by DCAF1E3-ubiquitin ligase [36-38]. Vpx interacts with the Cterminus of SAMHD1 in order to facilitate this degradation $[14,39,40]$. Recent reports have examined the acute kinetics of Vpx-mediated SAMHD1 degradation in myeloid cells and the enhancement of HIV-1 infection after Vpx treatment $[8,41]$. Further, we have reported in detail the acute effects of Vpx-mediated SAMHD1 degradation in monocyte-derived macrophages (MDMs) [42], which led to increased dNTP levels followed by enhancement of proviral DNA synthesis and transduction of MDMs.

In this report we performed an extensive kinetic and quantitative analysis examining the prolonged changes in dNTP concentrations and SAMHD1 levels over 14 days in primary human MDMs. In addition, we treated macrophages either with a single or dual VLP treatments, i.e. after the single Vpx + VLP treatment that kept SAMHD1 levels reduced by Western blots, and then measured dNTP levels and cellular nucleotide metabolites. While SAMHD1 remained very low, a second Vpx + VLP treatment promoted a rapid and robust increase in dNTPs. Collectively these data suggest that a very low level of SAMHD1 can dramatically modulate the dNTP concentrations in primary human MDMs.

\section{Results}

Monitoring the long-term kinetics of SAMHD1 levels and dNTP concentrations in human primary monocyte-derived macrophages

We previously reported the acute effects of Vpx containing virus-like particles (VLP) treatment on human primary MDMs out to $48 \mathrm{~h} \mathrm{[42]} \mathrm{and} \mathrm{observed} \mathrm{that} \mathrm{the} \mathrm{dGTP} \mathrm{levels}$ had peaked at day 1 and already started to decline at day 2 post Vpx treatment, while the SAMHD1 protein remained undetectable by western blot analysis. Many investigators have reported the effects of brief Vpx exposures in various cell types $[8,18,19,41-43]$. In this study, we investigated the recovery of SAMHD1 and changes in all four dNTP concentrations over 14 days in order to better understand cellular biology of SAMHD1 in primary human MDM treated with Vpx- or Vpx + VLP. As shown in Figure 1A, SAMHD1 remained undetectable under the immunoblot conditions described in previous studies until day 5 , which is consistent with the long half-life of Vpx [6]. Vpx has a $30 \mathrm{~h}$ half-life, suggesting it would be decayed by day 5 after VLP + VLP treatment. Quantitative analysis for the SAMHD1 protein level $(20 \mu \mathrm{g})$ from three donors (Figure 1B) revealed that at day 6, the SAMHD1 level was less than 3\% of the normal endogenous SAMHD1 level found in MDMs, which was confirmed by loading $3 \times(60 \mu \mathrm{g})$ the amount of protein at day 6 (Figure 1C). Importantly, SAMHD1 began to recover to a consistently detectable level around day 7 and continued to recover to only $30 \%$ expression at day 14 (Figure 1A). These data indicate that a single treatment of Vpx + VLP can induce a very prolonged phase of SAMHD1 reduction in MDMs followed by a slow recovery of protein.

Next, we employing our highly sensitive HIV-1 RTbased dNTP assay [44] to examine the effects on dNTP pools after Vpx-mediated SAMHD1 degradation in the same MDM donors used in Figure 1B. As shown in Figures 1D-G, increases in all four dNTPs were clearly detected after Vpx + VLP treatment in MDMs. dATP and dGTP concentrations (Figures $1 \mathrm{D}$ and $1 \mathrm{E}$ ) were significantly increased, while dCTP and dTTP concentrations (Figures $1 \mathrm{~F}$ and $1 \mathrm{G}$ ) were only modestly increased. dGTP concentration peaked at day 1 , consistent with our published results [42]. Importantly, dATP peaked at day 2 before the contraction began, indicating dNTP modulation was different for each of the nucleotides. The dNTP contraction occurred even though SAMHD1 protein remained undetectable at days $3-5$ by Western blot analysis. Basically, the rapid dNTP retraction following its acute elevation by Vpx occurred much ahead of the SAMHD1 recovery. These data suggest that the decrease in dNTP levels may be independent of the total SAMHD1 protein level due to various possibilities, which are discussed below.

Effect of dual Vpx + VLP treatment on dNTP levels in MDMs The day 7 time point after single Vpx + VLP treatment of MDMs (Figure 1B) provided us with the unique opportunity to test whether dual $(2 \times) \mathrm{Vpx}+\mathrm{VLP}$ treatment also influences the dNTP concentrations when SAMHD1 was less than $5 \%$ of the normal SAMHD1 level. For this test, dual Vpx + VLP treatment was performed at day 7 post primary VLP treatment and samples were collected every $24 \mathrm{~h}$ from days 7-14 (Additional file 1). We speculated that the peak in dNTP production during the dual Vpx + VLP treatment could have been missed while conducting kinetics analysis at $24 \mathrm{~h}$ time points. Therefore, as depicted in Figure 2A, we performed tighter acute kinetic dNTP analysis starting at day 7 after the dual $\mathrm{Vpx}+\mathrm{VLP}$ treatment in the MDMs and looking within 


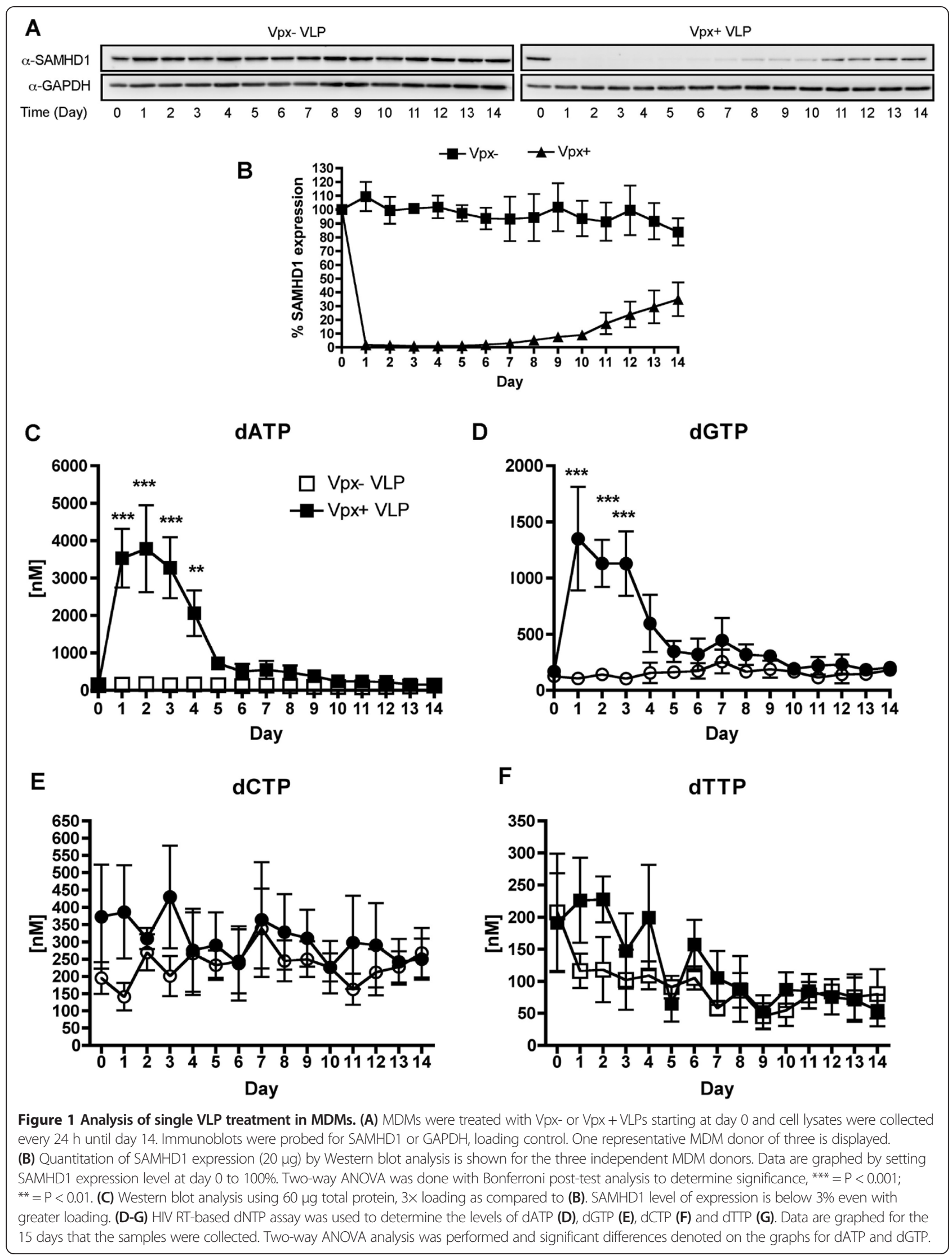




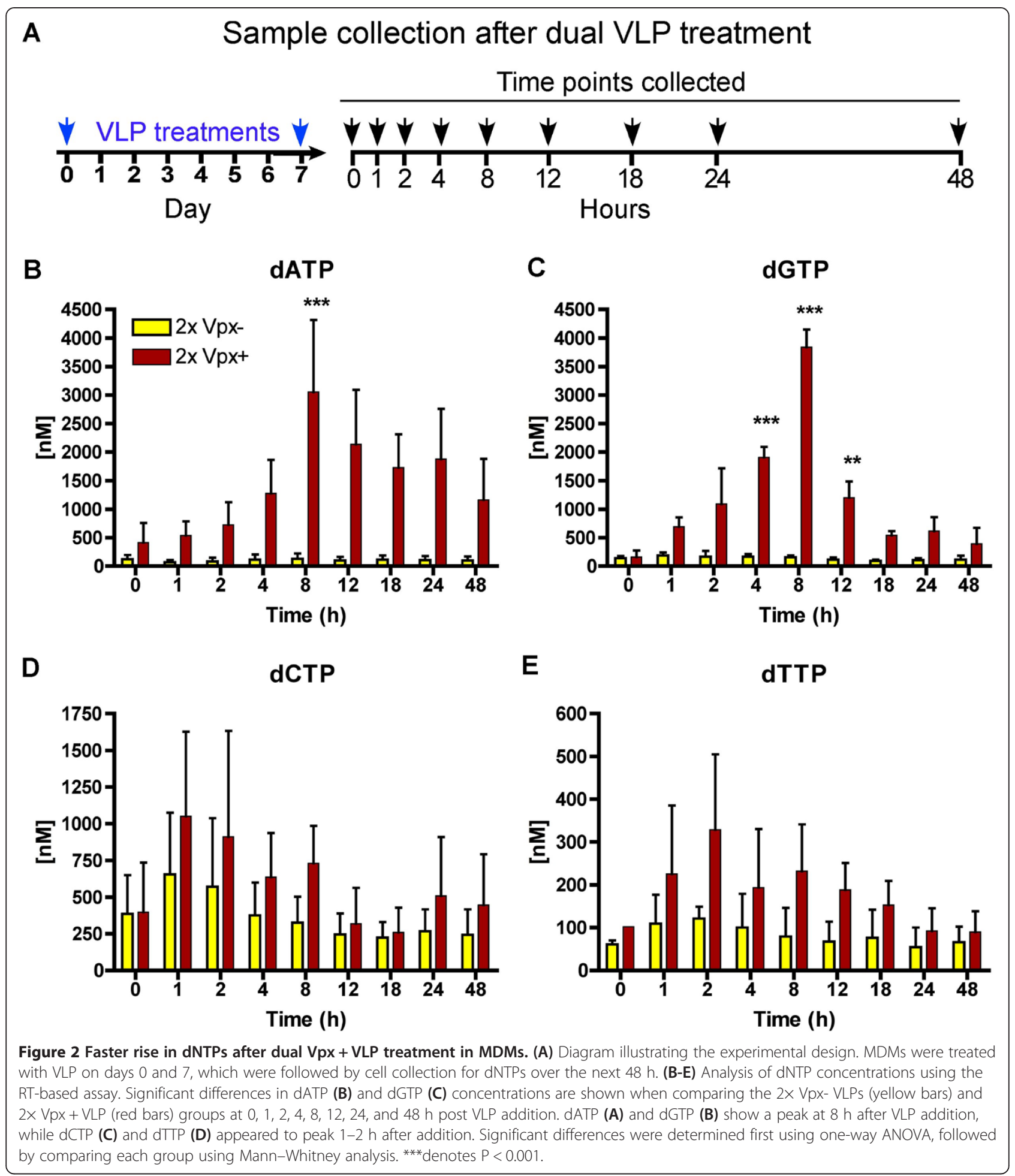

hours after dual treatment. As shown in Figures 2B-E, the second Vpx + VLP treatment showed an accelerated $\mathrm{dNTP}$ enhancement that peaked at around $8 \mathrm{~h}$ for dATP (Figure 2B) and dGTP (Figure 2C). Even more surprisingly, dCTP (Figure 2D) and dTTP (Figure 2E) peaked at
1-2 $\mathrm{h}$ post VLP treatment. Basically, the dual Vpx + VLP treated MDMs produced an accelerated increase in dNTP concentrations as compared to the single Vpx + VLP treatment [42]. Several possible scenarios may explain the faster dNTP elevation induced by the second Vpx + VLP 
treatment. First, in addition to its ability to promote SAMHD1 degradation, we speculate that Vpx may also activate the overall dNTP biosynthesis pathways. Alternatively, ribonucleotide reductase (RNR) activity through feedback modulation by cellular dATP was somehow changed from the primary and second Vpx + VLP treatment. This would help account for the fairly rapid contraction in dNTP concentrations after the second Vpx + VLP treatment. We did not see any modulation in the subunits of RNR after Vpx + VLP treatment (Additional file 2), but we have not been able to rule out Vpx potential modulation of RNR activity by an unknown mechanism. Another potential mechanism is that Vpx may target a putative minor SAMHD1 population that may be phosphorylated. Preliminary data suggest that MDMs can have a PSAMHD1 at T592 population, but both time in culture and SAMHD1 protein recovery after Vpx + VLP treatment showed the loss and the lack of phosphorylated SAMHD1, respectively (Additional file 3). This rules out modulation of dNTP concentrations by SAMHD1 phosphorylation during the recovery phase of SAMHD1 (days 7-14).

\section{Comparison of the effects of the single and dual Vpx + VLP treatments on HIV-1 transduction efficiency in MDMs}

Next, we investigated how single and dual Vpx + VLP treatments affects HIV-1 transduction in MDMs. For this, we employed D3HIV-GFP vector that encodes the entire HIV-1 genome except for env and nef, which are deleted and replaced with $e G F P$. As shown in Figure 3A, single VLP treatment and D3HIV-GFP vector was added to the medium at day 0 , followed by collection of the MDMs at days 1, 2 and 7 for FACS analysis. As illustrated in Figure 3B, MDMs were treated at days 0 and 7 with VLPs, and then D3HIV-GFP vector was added at day 7. MDMs were collected at days 8, 9 and 14 (corresponding to days 1, 2 and 7 after D3HIV-GFP vector addition). The vector transduction efficiency was measured by monitoring eGFP expression by FACS analysis from two independent MDM donors and graphed at days 1, 2 and 7 post D3HIV-GFP vector transduction (Figures $3 \mathrm{C}$ and 3D). MDMs treated with Vpx- VLP and D3HIV-GFP had about $5 \%$ transduction at day 7 post transduction (see white bars in Figure $3 \mathrm{C}$ and $3 \mathrm{D})$. The single Vpx + VLP treatment (black bars in Figure $3 \mathrm{C}$ and $3 \mathrm{D}$ ) enhanced HIV-1 vector transduction to $\sim 30 \%$ transduction at day 7 as compared to the Vpx- VLP treatment ( $5 \%$ at day 7$)$. However, the dual Vpx + VLP treatment (red bars) was able to induce $50-60 \%$ transduction at day 7 as compared to the single Vpx + VLP treatment (black bars). Collectively, these data support that the faster dNTP increase by the dual Vpx + VLP treatment was able to further enhance HIV-1 transduction in MDMs.

\section{Measurement of dNTP intermediate metabolites by quantitative LC-MS/MS}

The overall dNTP biosynthesis pathways has more intermediates than what our HIV RT-based dNTP assay can measure. Since we observed a faster dNTP elevation by the dual Vpx + VLP treatment as compared to the single Vpx + VLP treatment (Figure 2), and that the rapid dNTP retraction observed before any detectable SAMHD1 reappears (Figure 1), we monitored the metabolic changes in all three dNTP intermediate precursors: deoxynucleoside monophosphates (dNMPs) and diphosphates (dNDPs) and triphosphates (dNTPs) metabolites, by employing quantitative LC-MS/MS technology [45]. For these studies MDMs were treated with either single VLP doses and cells were harvested for dNTPs $24 \mathrm{~h}$ later. For the dNTP levels (Figure 4A), it is clear that the single Vpx + VLP treatment enhances the levels of and at least dADP, dGDP and dCDP, while dTDP was below the level of detection (see \# in Figure 4B). But the dual Vpx + VLP treatment (Figure 4D-F) also induced greater dNDP levels, particularly purine $\mathrm{dNDP}$ as compared to the same MDM donor treated with Vpx- VLP. For dNMPs levels, only the dAMP measurement generated significantly detectable signals in both single Vpx + VLP (Figure 4C) and dual Vpx + VLP (Figure 4F) treatments in MDMs. Importantly, the LC-MS/MS analysis supports that Vpx + VLP treatment not only elevates the dNTP levels in MDMs but also the dNTP intermediate precursors. Since the medium lacks deoxyribonucleosides, we postulate via indirect evidence that the increase in dNDP metabolites has to occur through the activation of RNR. The exact mechanism remains unclear and is not linked to an increase in RNR subunit protein levels (Additional file 2). Moreover, $\mathrm{Vpx}$ is localized to the nucleus [5,10,14-19], suggesting that it does not have a direct interaction with RNR.

\section{Effect of gemcitabine, an RNR inhibitor, on the Vpx-mediated dNTP elevation in MDMs}

It was previously demonstrated that hydroxyurea, an RNR inhibitor, could block Vpx-mediated increase in dNTPs [41]. We examined the contribution of RNR to increase the dNTP concentrations after Vpx-mediated SAMHD1 degradation in MDMs. We first tested dose-escalating concentrations of gemcitabine, a clinically available RNR inhibitor [46], for 24 h (Additional file 4). From these data, we tested 40 and $100 \mathrm{nM}$ dose-escalating concentrations of gemcitabine. MDMs were pretreated $22.5 \mathrm{~h}$ with VLPs before adding gemcitabine. Cells were collected $1.5 \mathrm{~h}$ later and analyzed for changes in dNTP levels (Figure 5). We observe a $50 \%$ reduction in the levels of dATP (Figure $5 \mathrm{~A}$ ), dGTP (Figure 5B) and dCTP (Figure 5C) after $100 \mathrm{nM}$ gemcitabine treatments, whereas dTTP concentration (Figure 5D) was less influenced. Collectively, these data 


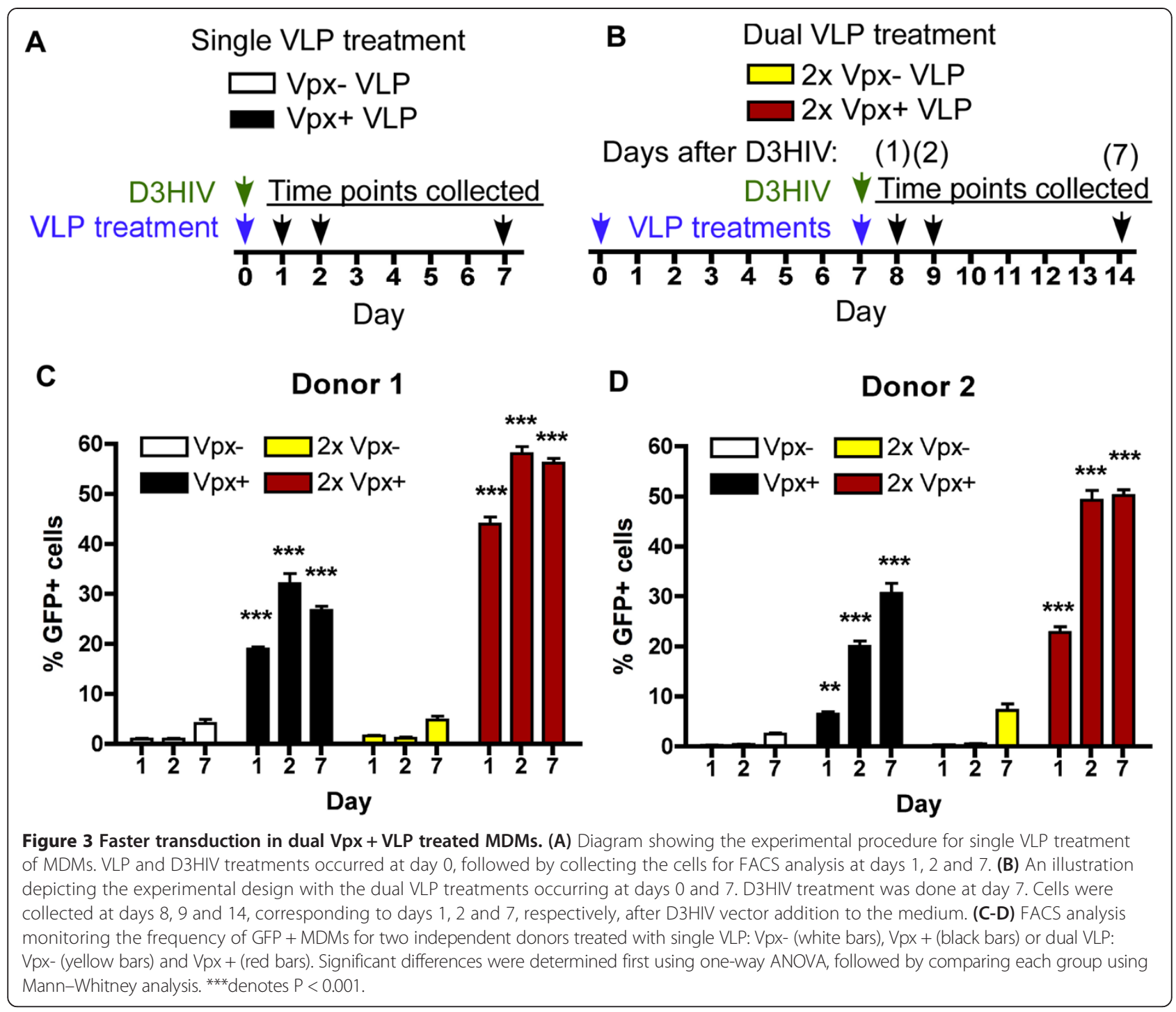

indicate that RNR greatly contributes to the overall increase in Vpx-mediated dNTPs increase in the absence of SAMHD1.

\section{Discussion}

This investigation began by examining how quickly SAMHD1 protein levels return after a single Vpx + VLP treatment in seven day maturated, primary human MDMs. We found that the level of SAMHD1 remained very low (less than $3 \%$ of the normal level in macrophages) between days 1-6 before it became consistently detectable by Western blot analysis at day 7. Vpx has been shown to have a long cellular half-life [6] and should be degraded roughly by day 5 after VLP treatment. However, SAMHD1 never recovered to its normal high level even at day 14, suggesting de novo protein synthesis of SAMHD1 may also be very slow or negatively regulated after Vpx treatment. Examining dNTP levels over this long time course showed a different observation. The levels of dNTPs were reduced well before the detection of SAMHD1 level by Western blot (Figure 1A), yet dNTPs declined starting at day 2 for dGTP, dCTP and dTTP and day 3 for dATP post Vpx + VLP treatment. Thus we speculate that additional factors or very low levels of SAMHD1 may be regulating dNTP pool sizes. We expected that the reduced level of SAMHD1 at day 7 post Vpx + VLP treatment would provide a window of opportunity to investigate cellular dNTP metabolism during a second Vpx + VLP exposure. Indeed, the dual Vpx + VLP treatment of MDMs at day 7 was able to display the same robust dNTP elevation at the time point when SAMHD1 remained less than $3 \%$ of the normal level in MDMs (Figure 2). We speculate that since the kinetics of the dual treatment are faster and the HLPC-MS data indicated an increase in dNDP metabolites, Vpx may harbor a SAMHD1 independent 


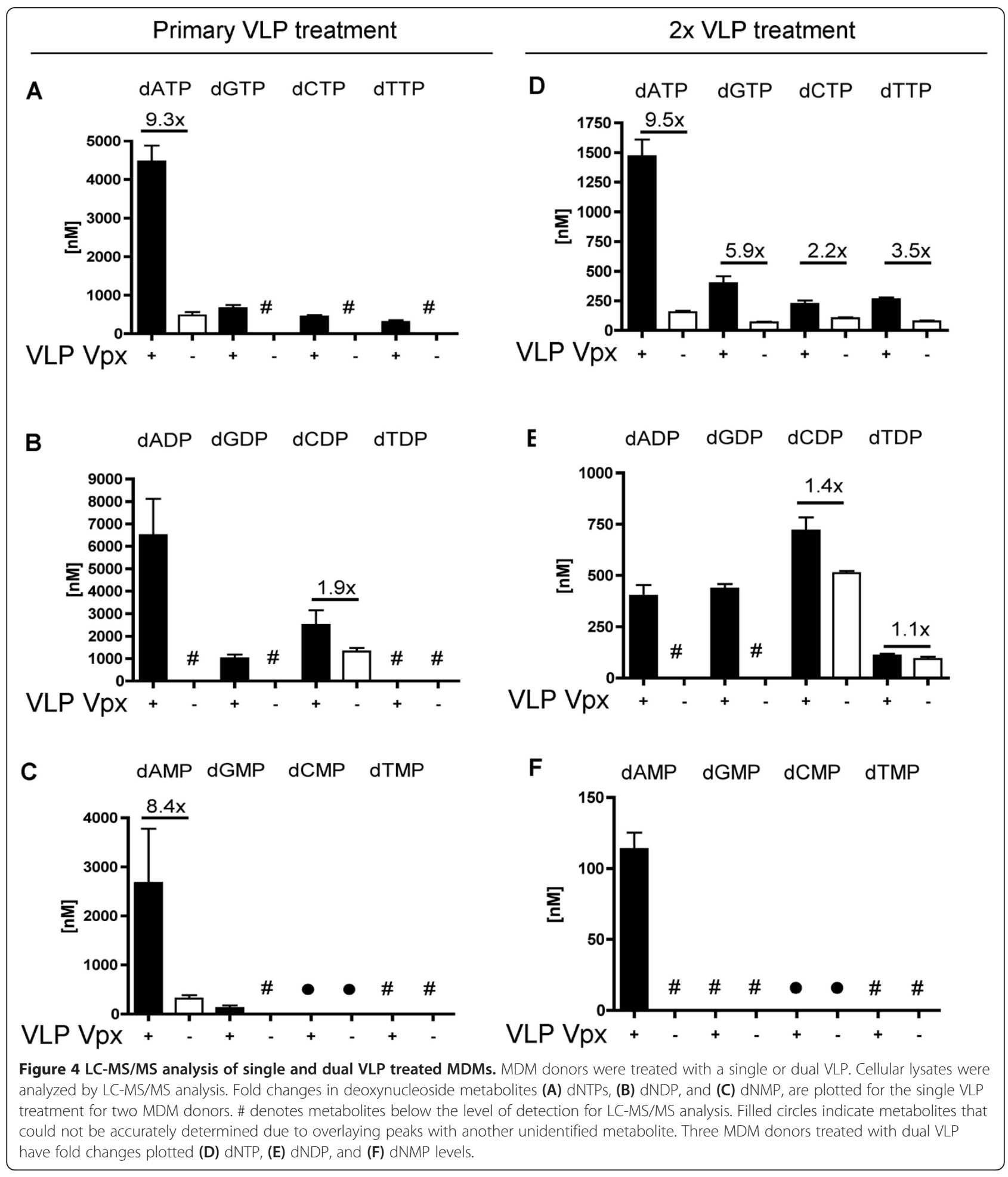

function that actively facilitates cellular dNTP biosynthesis metabolism and elevates cellular dNTP levels in the presence of only $3 \%$ of the normal SAMHD1 level. This potential mechanism is not related to the up regulations of RNR subunits (Additional file 2). Moreover, we have no direct evidence that $\mathrm{Vpx}$ interacts with any of the RNR subunits. Thus, it remains unclear as to just how Vpx VLP treatment and dNDP increase might be occurring.

Interestingly, the deoxypurine triphosphate (dATP and dGTP) concentrations remained high for several days after the single VLP treatment, while deoxypyrimidines - dCTP and dTTP concentrations showed 


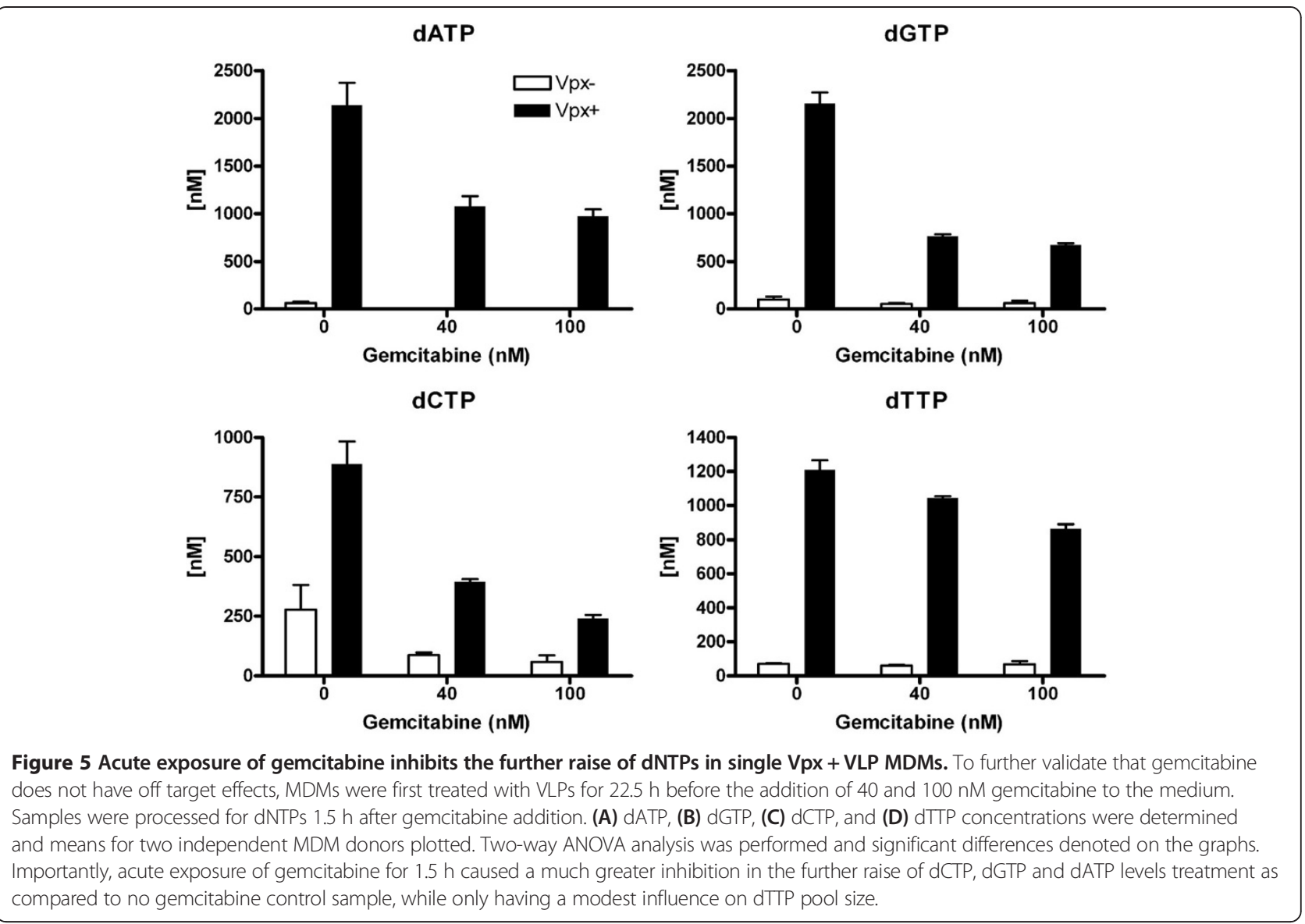

only a transient increase before returning to base line levels for the majority of the MDM donors tested in this study (Figures 1E-F). Our data are consistent with the recently generated SAMHD1 deficient mouse [5], which shows that dATP and dGTP concentrations were significantly increased in the SAMHD1 deficient mice as compared to wild type mice. Importantly, the dual Vpx + VLP treatment of MDMs was informative by showing that the peak of the dATP and dGTP occurred around $8 \mathrm{~h}$ post Vpx + VLP addition, which is much faster than our results published for dGTP on the acute kinetics by the single Vpx + VLP treatment [42]. Interestingly, we observed a decline in the levels of all dNTPs at day 3 post Vpx + VLP treatment, suggesting that turnover of the dNTP pool occurs by other underlying mechanisms other than SAMHD1. These other mechanisms may include hydrolysis by deoxynucleoside diphosphatases $[47,48]$, shut-off of RNR activity, or conversion to energy currency for other cellular enzyme reactions. Since MDMs are non-dividing cells, we can rule out that the decrease in dNTPs after a dual Vpx + VLP treatment is due to the dNTP utilization during DNA replication. However, we cannot rule out that DNA repair activity is occurring and is consuming the dNTPs. We postulate that the elimination of SAMHD1 may lead to establishing a new modulation set point of dNTP pools within the cell, with dATP and dGTP concentrations remaining much higher than dCTP and dTTP levels [5].

\section{Conclusions}

We employed a series of biochemical and virological investigations with extensive and multiple exposures of Vpx + VLP treatments to human primary MDMs. These studies revealed that there was a significant quantitative discord between levels of total SAMHD1 protein and cellular dNTPs when MDMs were treated with Vpx + VLP. One potential explanation is that Vpx may promote targeting of SAMHD1 for degradation but also facilitate dNTP biosynthesis in macrophages since we detect an increase in dNDP metabolites, which are the precursors for dNTPs. This in turn would achieve a rapid and robust dNTP elevation, which is necessary for accelerating lentiviral reverse transcription for HIV-2 and SIV and also DNA gap-filling repair as part of lentiviral integration [49], in cells having extremely low cellular dNTP abundance. 


\section{Methods}

\section{Ethics statement}

These experiments used primary human monocytes obtained from human buffy coats (New York Blood Services, Long Island, NY). These are pre-existing materials that are publicly available, and there is no subjectidentifying information associated with the material obtained from this supplier. As such, the use of these samples does not represent human subjects research because: 1) materials were not collected specifically for this study, and 2) we are not able to identify the subjects.

\section{Cells}

Primary human monocytes were isolated from the peripheral blood buffy coats by positive selection using MACS CD14+ beads as previously described [50]. Monocytes were maturated into monocyte-derived macrophages (MDMs) in the presence of $5 \mathrm{ng} / \mathrm{ml}$ hGM-CSF (Miltenyl Biotec) treated at days 0 and 2 of maturation. MDMs were used at day 7 of maturation for experiments.

\section{Primer extension assay}

Protocol was followed as previously described [42]. MDMs were lysed with $60 \%$ cold methanol. Cellular debris was cleared by $14 \mathrm{~K}$ rpm centrifugation. Supernatant was dried using a SpeedVac (Thermo Scientific). Pellets were resuspended in $20 \mu \mathrm{l}$ water. Two microliters of sample were used in the primer extension assay. $5{ }^{32} \mathrm{P}$-endlabeled primer ("P”; 5'-GTCCCTCTTCGGGCGCCA-3') was individually annealed to one of four different templates (3'-CAGGGAGAAGCCCGCGGTN-5'). The template:primer complex was extended by $\mathrm{HIV}-1$ reverse transcriptase, generating one additional nucleotide extension product ("P +1 ") for one of four dNTPs contained in the dNTP samples extracted form the cells. In this assay, the molar amount of the $\mathrm{P}+1$ product is equal to that of each dNTP contained in the extracted samples, which allows us to calculate and compare the dNTP concentrations for the different treatments [44].

\section{VLP generation}

T225 flasks containing 293FT cells were transfected with $40 \mu \mathrm{g}$ of pVpx- VLP or pVpx + VLP (kindly provided by Drs. Florence Margottin-Goguet and Nathaniel Landau) and $20 \mu \mathrm{g}$ of pVSVg at a ratio of $1 \mu \mathrm{g}$ of DNA to $3 \mu \mathrm{l}$ of polyethylenimine $(1 \mathrm{mg} / \mathrm{ml})$. The following day, medium was discarded and replaced with fresh DMEM medium (5\% FBS and antibiotics). On days 2-3 after transfection, the medium was collected and replaced with fresh medium. On the day of collection, medium was centrifuged at $1200 \mathrm{rpm}$ for $5 \mathrm{~min}$ to remove cells. Supernatant was subsequently filtered through a $0.45-\mu \mathrm{m}$ membrane (Corning Inc.) and overlaid on top of $5 \mathrm{ml}$ of a $25 \%$ sucrose cushion $(25 \%(\mathrm{w} / \mathrm{v})$ sucrose, $10 \mathrm{mM}$ Tris- $\mathrm{HCl}$
[pH 7.5], $0.1 \mathrm{M} \mathrm{NaCl}$ and $1 \mathrm{mM}$ EDTA). VLPs were concentrated at $28,000 \mathrm{rpm}$ for $90 \mathrm{~min}$ by ultracentrifugation. Supernatant was aspirated, and pellets were resuspended in $600 \mu \mathrm{l}$ of serum-free DMEM. Supernatant was centrifuged for $1 \mathrm{~min}$ at $14 \mathrm{~K} \mathrm{rpm}$ to remove debris. Aliquots $(50 \mu \mathrm{l})$ were stored at $-80^{\circ} \mathrm{C}$. The p27 antigen level was determined using an ELISA kit (Advanced BioScience Laboratories, Inc., Rockville MD). A minimum of $145 \mathrm{ng}$ of $\mathrm{p} 27 /$ million cells was used in experiments.

\section{D3HIV-GFP generation}

pD3HIV-GFP vector encodes the HIV-1 NL4-3 genome with the eGFP gene in place of the HIV-1 nef gene and has a deleted envelope gene [44]. To generate virus, 293FT cells in T225 flasks were transfected with $60 \mu \mathrm{g}$ pD3HIV-GFP and $20 \mu \mathrm{g}$ pVSV-g using $140 \mu \mathrm{l}$ polyethyenimine $(1 \mathrm{mg} / \mathrm{ml})$ in $37 \mathrm{ml}$ DMEM medium/flask. At day 1 of HIV-1 production, medium was discarded and replaced with fresh complete DMEM medium (5\% FBS plus antibiotics). At day 2, the medium was harvested and replaced. The medium was centrifuged at $2500 \mathrm{rpm}$ for $7 \mathrm{~min}$ to remove cellular debris, and then stored at $4^{\circ} \mathrm{C}$ in T75 flask. Day 3 medium was harvested and processed as described for day 2. D3HIV-GFP was concentrated using ultracentrifugation $(22 \mathrm{~K} \mathrm{rpm}$ for $2 \mathrm{~h}$ in a SW32 Ti rotor). Pellets were resuspended in $0.5 \mathrm{ml}$ serum free DMEM medium. Afterwards, debris was removed by centrifugation ( $14 \mathrm{~K}$ for $2 \mathrm{~min})$. Sample aliquots $(50 \mu \mathrm{l})$ were frozen at $-80^{\circ} \mathrm{C}$ until used. MDMs were transduced with D3HIV-GFP and then the samples were analyzed using Accuri C6 flow cytometer monitoring GFP expression at the indicated times. Data files were analyzed using FlowJo software (TreeStar).

\section{Mass spectrometry}

MDM extracts were generated by scraping wells with $70 \%$ methanol and freezing them overnight at $-80^{\circ} \mathrm{C}$. Extracts were centrifuged at $13,000 \times g$ for $3 \mathrm{~min}$ and the supernatants were subsequently dried. The resulting samples were reconstituted in HPLC mobile phase for LC-MS/MS analysis as described previously [51]. In short, samples were reconstituted in $200 \mu \mathrm{l}$ of $2 \mathrm{mM}$ $\mathrm{NH}_{4} \mathrm{H}_{2} \mathrm{PO}_{4}$ with $3 \mathrm{mM}$ hexylamine then analyzed for deoxyribonucleosides. Samples were separated using Hypersil Gold $100 \times 1 \mathrm{~mm}$ column using Mobile phase - A: acetonitrile and B: $2 \mathrm{mM} \mathrm{NH}_{4} \mathrm{H}_{2} \mathrm{PO}_{4}$ with $3 \mathrm{mM}$ hexylamine. A increased from $5 \%$ to $50 \%$ in $10 \mathrm{~min}$, keep $50 \%$ for $3 \mathrm{~min}$. The $m / z$ parent to product MS/MS transitions: 523 to 146 , 539 to 162,496 to 119 , and 495 to 81 were applied for the standard stable labeled isotopes and 508 to 136,524 to 152 , 484 to 112 , and 485 to 81 for the corresponding sample nucleotides, respectively.

Samples were reconstituted in $200 \mu \mathrm{l}$ of $2 \mathrm{mM}$ $\mathrm{NH}_{4} \mathrm{H}_{2} \mathrm{PO}_{4}$ with $3 \mathrm{mM}$ hexylamine, and then split into 
two fractions. One fraction was analysis for deoxyribonucleosides. Samples were separated using Hypersil Gold $100 \times 1 \mathrm{~mm}$ column using Mobile phase - A: acetonitrile and B: $2 \mathrm{mM} \mathrm{NH}_{4} \mathrm{H}_{2} \mathrm{PO}_{4}$ with $3 \mathrm{mM}$ hexylamine. A increased from $5 \%$ to $50 \%$ in $10 \mathrm{~min}$, keep $50 \%$ for $3 \mathrm{~min}$. Instrument parameters were optimized for each metabolite (Additional file 5).

\section{Western blot analysis}

Samples were processed in RIPA buffer containing $1 \mu \mathrm{M}$ DTT, $10 \mu \mathrm{M}$ PMSF, $10 \mu \mathrm{l} / \mathrm{ml}$ phosphatase inhibitor (Sigma) and $10 \mu \mathrm{l} / \mathrm{ml}$ protease inhibitor (Sigma). The cells were sonicated with $3 \times, 5$ second pulses, to ensure complete lysis. Cellular debris was removed by $15 \mathrm{~K} \mathrm{rpm}$ centrifugation for $10 \mathrm{~min}$. Supernatants were stored at $-80^{\circ} \mathrm{C}$ before use. Cell lysates were resolved on a $8 \%$ SDS-PAGE gel. Proteins were transferred to nitrocellulose membrane and detected as described in the figure legends using the following antibodies: rabbit anti-SAMHD1 mAb (Abcam), anti-GAPDH mouse mAb (Santa Cruz). Anti-mouse and anti-rabbit secondary HRP antibodies were purchased from GE HealthScience. HRP was detected using chemiluminescent reagents (Pierce) following the manufacturers instructions. Images were captured using BioRad ChemiDoc Imager. Anti-pSAMHD1 T592 antibody was obtained from Dr. Diaz-Griffero.

\section{Graphing and statistical analysis}

Prism software was used for plotting the data. All the data sets were compared for significant difference using two-way ANOVA analysis and Bonferroni post-test analysis for significance.

\section{Additional files}

Additional file 1: Dual Vpx + VLP Kinetic Analysis. (A) Diagram showing the treatment regime for single and dual VLP treatments and the days harvested after treatment. (B-E) The HIV-1 RT-based dNTP assay was performed on samples to determine concentrations of the four different metabolites. Two-way- ANOVA was applied to determine significant differences within each group indicated $\left({ }^{*}, \mathrm{P}<0.05 ;{ }^{* *}, \mathrm{P}<0.01\right.$ and ***, $P<0.001$ ). Analysis was done for two independent MDM donors.

Additional file 2: Analysis of RNR subunits. MDMs were treated with VLP for $24 \mathrm{~h}$ and then processed for cellular lysates. Western blot analysis was performed using $25 \mu \mathrm{g}$ total protein to detect the major subunit, R1 (Abcam), or the small subunits R2 and p53R2 (Santa Cruz). No significant changes in RNR expression levels were detected.

Additional file 3: Analysis of pSAMHD1 at 592. Two independent MDM donors were examined for PSAMHD1 by immunoblot analysis (20 $\mu \mathrm{g}$ protein). Total SAMHD1 was determined and GAPDH was used as the internal loading control.

Additional file 4: Gencitabine inhibition of Vpx-mediated dNTP increase. MDMs were pretreated for $2 \mathrm{~h}$ with various concentration of gemcitabine as indicated in figures. Next, VLPs were added and cells were placed in the incubator for $24 \mathrm{~h}$, after which time they were processed for dNTPs. The HIV-1 RT-based dNTP assay was done and data plotted for each metabolite: (A) dATP, (B) dGTP, (C) dCTP and (D) dTTP. One-way ANOVA was done and significant differences indicated $\left({ }^{*}, \mathrm{P}<0.05 ;{ }^{* *}, \mathrm{P}<0.01\right.$ and ***, $P<0.001)$. Analysis was done for two independent donors.

Additional file 5: HPLC-MS settings for instrument optimization. Each metabolite was purchased from Sigma and then ran on the HLPC-MS to optimize the reading. Then setting were then used to generate a concentration curve that was applied to determine the concentrations of these metabolites in MDMs treated with Vpx + VLP and Vpx- VLPs.

\section{Competing interests}

The authors declare that they have no competing interests.

\section{Authors' contributions}

JAH and ST performed experiments. GML, SR, DK and RFS contributed conceptually to the experimental design. JAH, GML and BK wrote the manuscript. FDG provided reagents. All authors read and approved the final manuscript.

\section{Acknowledgements}

We would like to thank Waaqo Daddacha and Laura Nguyen for technical assistance with the HIV-1 RT-based dNTP assay. We would also like to thank Michael Hirschman and Michele Daly for technical assistance. This study was supported by NIH Al049781 (B.K.), GM104198 (B.K.), 5P30-Al-50409 Emory Centers for AIDS Research (CFAR), Al087390 (F. D-G), and the Department of Veterans Affairs.

\section{Author details}

'Department of Pediatrics, Center for Drug Discovery, Emory Center for AIDS Research, Laboratory of Biochemical Pharmacology, Emory University School of Medicine, 1760 Haygood Drive, Health Sciences Research Building, Atlanta, Georgia 30322, USA. ${ }^{2}$ Department of Microbiology and Immunology, Albert Einstein College of Medicine Bronx, Bronx, NY 10461, USA. ${ }^{3}$ Veterans Affairs Medical Center, Decatur, Georgia 30033, USA. ${ }^{4}$ College of Pharmacy, Kyung-Hee University, Seoul, South Korea.

Received: 23 April 2014 Accepted: 18 July 2014 Published: 27 August 2014

\section{References}

1. Rice GI, Bond J, Asipu A, Brunette RL, Manfield IW, Carr IM, Fuller JC, Jackson RM, Lamb T, Briggs TA, Ali M, Gornall H, Couthard LR, Aeby A, Attard-Montalto SP, Bertini E, Bodemer C, Brockmann K, Brueton LA, Corry PC, Desguerre I, Fazzi E, Cazorla AG, Gener B, Hamel BC, Heiberg A, Hunter M, van der Knaap MS, Kumar R, Lagae L, et al: Mutations involved in Aicardi-Goutieres Syndrome Implicate Samhd1 as Regulator of the Innate Immune Response. Nat Genet 2009, 41:829-832.

2. Kretschmer S, Wolf C, Konig N, Staroske W, Guck J, Hausler M, Luksch H, Nguyen LA, Kim B, Alexopoulou D, Dahl A, Rapp A, Cardoso MC, Shevchenko A, Lee-Kirsch MA: Samhd1 Prevents Autoimmunity by Maintaining Genome Stability. Ann Rheum Dis 2014.

3. Ramantani G, Kohlhase J, Hertzberg C, Innes AM, Engel K, Hunger S, Borozdin W, Mah JK, Ungerath K, Walkenhorst H, Richardt HH, Buckard J, Bevot A, Siegel C, von Stulpnagel C, Ikonomidou C, Thomas K, Proud V, Niemann F, Wieczorek D, Hausler M, Niggemann P, Baltaci V, Conrad K, Lebon P, Lee-Kirsch MA: Expanding the Phenotypic Spectrum of Lupus Erythematosus in Aicardi-Goutieres Syndrome. Arthritis Rheum 2010, 62:1469-1477.

4. Lee-Kirsch MA: Nucleic Acid Metabolism and Systemic Autoimmunity Revisited. Arthritis Rheum 2010, 62:1208-1212.

5. Behrendt R, Schumann T, Gerbaulet A, Nguyen LA, Schubert N, Alexopoulou D, Berka U, Lienenklaus S, Peschke K, Gibbert K, Wittmann S, Lindemann D, Weiss S, Dahl A, Naumann R, Dittmer U, Kim B, Mueller W, Gramberg T, Roers A: Mouse Samhd1 Has Antiretroviral Activity and Suppresses a Spontaneous Cell-Intrinsic Antiviral Response. Cell Rep 2013, 4:689-696.

6. Berger G, Turpin J, Cordeil S, Tartour K, Nguyen XN, Mahieux R, Cimarelli A: Functional Analysis of the Relationship between Vpx and the Restriction Factor Samhd1. J Biol Chem 2012, 287:41210-41217.

7. Dragin L, Nguyen LA, Lahouassa H, Sourisce A, Kim B, Ramirez BC, Margottin-Goguet F: Interferon Block to Hiv-1 Transduction in Macrophages Despite Samhd1 Degradation and High Deoxynucleoside Triphosphates Supply. Retrovirology 2013, 10:30. 
8. Hrecka K, Hao C, Gierszewska M, Swanson SK, Kesik-Brodacka M, Srivastava S, Florens L, Washburn MP, Skowronski J: Vpx Relieves Inhibition of Hiv-1 Infection of Macrophages Mediated by the Samhd1 Protein. Nature 2011, 474:658-661.

9. Mashiba M, Collins KL: Molecular Mechanisms of Hiv Immune Evasion of the Innate Immune Response in Myeloid Cells. Viruses 2013, 5:1-14.

10. Rehwinkel J, Maelfait J, Bridgeman A, Rigby R, Hayward B, Liberatore RA, Bieniasz PD, Towers GJ, Moita LF, Crow YJ, Bonthron DT, Reis ESC: Samhd1-Dependent Retroviral Control and Escape in Mice. EMBO J 2013, 32:2454-2462

11. White TE, Brandariz-Nunez A, Valle-Casuso JC, Amie S, Nguyen LA, Kim B, Tuzova M, Diaz-Griffero F: The Retroviral Restriction Ability of Samhd1, but Not Its Deoxynucleotide Triphosphohydrolase Activity, Is Regulated by Phosphorylation. Cell Host Microbe 2013, 13:441-451.

12. Yu H, Usmani SM, Borch A, Kramer J, Sturzel CM, Khalid M, Li X, Krnavek D, van der Ende ME, Osterhaus AD, Gruters RA, Kirchhoff F: The Efficiency of Vpx-Mediated Samhd1 Antagonism Does Not Correlate with the Potency of Viral Control in Hiv-2-Infected Individuals. Retrovirology 2013, 10:27.

13. Clifford R, Louis T, Robbe P, Ackroyd S, Burns A, Timbs AT, Wright Colopy G, Dreau H, Sigaux F, Judde JG, Rotger M, Telenti A, Lin YL, Pasero P, Maelfait J, Titsias M, Cohen DR, Henderson SJ, Ross MT, Bentley D, Hillmen P, Pettitt A, Rehwinkel J, Knight SJ, Taylor JC, Crow YJ, Benkirane M, Schuh A: Samhd1 Is Mutated Recurrently in Chronic Lymphocytic Leukemia and Is Involved in Response to DNA Damage. Blood 2014, 123:1021-1031.

14. Wei W, Guo H, Han X, Liu X, Zhou X, Zhang W, Yu XF: A Novel Dcaf1-Binding Motif Required for Vpx-Mediated Degradation of Nuclear Samhd1 and Vpr-Induced G2 Arrest. Cell Microbiol 2012, 14:1745-1756.

15. Hofmann H, Logue EC, Bloch N, Daddacha W, Polsky SB, Schultz ML, Kim B, Landau NR: The Vpx Lentiviral Accessory Protein Targets Samhd1 for Degradation in the Nucleus. J Virol 2012, 86:12552-12560.

16. Schaller T, Pollpeter D, Apolonia L, Goujon C, Malim MH: Nuclear Import of Samhd1 Is Mediated by a Classical Karyopherin Alpha/Beta1 Dependent Pathway and Confers Sensitivity to Vpxmac Induced Ubiquitination and Proteasomal Degradation. Retrovirology 2014, 11:29.

17. Brandariz-Nunez A, Valle-Casuso JC, White TE, Laguette N, Benkirane M, Brojatsch J, Diaz-Griffero F: Role of Samhd1 Nuclear Localization in Restriction of Hiv-1 and Sivmac. Retrovirology 2012, 9:49.

18. Baldauf HM, Pan X, Erikson E, Schmidt S, Daddacha W, Burggraf M, Schenkova K, Ambiel I, Wabnitz G, Gramberg T, Panitz S, Flory E, Landau NR, Sertel S, Rutsch F, Lasitschka F, Kim B, Konig R, Fackler OT, Keppler OT: Samhd1 Restricts Hiv-1 Infection in Resting Cd4(+) T Cells. Nat Med 2012, 18:1682-1689.

19. St Gelais C, de Silva S, Amie SM, Coleman CM, Hoy H, Hollenbaugh JA, Kim B, Wu L: Samhd1 Restricts Hiv-1 Infection in Dendritic Cells (Dcs) by Dntp Depletion, but Its Expression in Dcs and Primary Cd4+ T-Lymphocytes Cannot Be Upregulated by Interferons. Retrovirology 2012, 9:105.

20. Goldstone DC, Ennis-Adeniran V, Hedden JJ, Groom HC, Rice Gl, Christodoulou E, Walker PA, Kelly G, Haire LF, Yap MW, de Carvalho LP, Stoye JP, Crow YJ, Taylor IA, Webb M: Hiv-1 Restriction Factor Samhd1 Is a Deoxynucleoside Triphosphate Triphosphohydrolase. Nature 2011, 480:379-382.

21. Powell RD, Holland PJ, Hollis T, Perrino FW: The Aicardi-Goutieres Syndrome Gene and Hiv-1 Restriction Factor Samhd1 Is a Dgtp-Regulated Deoxynucleotide Triphosphohydrolase. J Biol Chem 2011, 286:43596-43600.

22. Sze A, Belgnaoui SM, Olagnier D, Lin R, Hiscott J, van Grevenynghe J: Host Restriction Factor Samhd1 Limits Human T Cell Leukemia Virus Type 1 Infection of Monocytes Via Sting-Mediated Apoptosis. Cell Host Microbe 2013, 14:422-434.

23. Kim ET, White TE, Brandariz-Nunez A, Diaz-Griffero F, Weitzman MD: Samhd1 Restricts Herpes Simplex Virus 1 in Macrophages by Limiting DNA Replication. J Virol 2013, 87:12949-12956

24. Hollenbaugh JA, Gee P, Baker J, Daly MB, Amie SM, Tate J, Kasai N, Kanemura Y, Kim DH, Ward BM, Koyanagi Y, Kim B: Host Factor Samhd1 Restricts DNA Viruses in Non-Dividing Myeloid Cells. PLoS Pathog 2013, 9:e1003481.

25. White TE, Brandariz-Nunez A, Carlos Valle-Casuso J, Amie S, Nguyen L, Kim B, Brojatsch J, Diaz-Griffero F: Contribution of Sam and Hd Domains to Retroviral Restriction Mediated by Human Samhd1. Virology 2012, 436:81-90.

26. Zhu C, Gao W, Zhao K, Qin X, Zhang Y, Peng X, Zhang L, Dong Y, Zhang W, Li P, Wei W, Gong Y, Yu XF: Structural Insight into Dgtp-Dependent Activation of Tetrameric Samhd1 Deoxynucleoside Triphosphate Triphosphohydrolase. Nat Commun 2013, 4:2722.

27. Yan J, Kaur S, DeLucia M, Hao C, Mehrens J, Wang C, Golczak M, Palczewski K, Gronenborn AM, Ahn J, Skowronski J: Tetramerization of Samhd1 Is Required for Biological Activity and Inhibition of Hiv Infection. J Biol Chem 2013, 288:10406-10417.

28. Ji X, Wu Y, Yan J, Mehrens J, Yang H, DeLucia M, Hao C, Gronenborn AM, Skowronski J, Ahn J, Xiong Y: Mechanism of Allosteric Activation of Samhd1 by Dgtp. Nat Struct Mol Biol 2013, 20:1304-1309.

29. Brandariz-Nunez A, Valle-Casuso JC, White TE, Nguyen L, Bhattacharya A, Wang Z, Demeler B, Amie S, Knowlton C, Kim B, Ivanov DN, Diaz-Griffero F: Contribution of Oligomerization to the Anti-Hiv-1 Properties of Samhd1. Retrovirology 2013, 10:131.

30. Hansen EC, Seamon KJ, Cravens SL, Stivers JT: Gtp Activator and Dntp Substrates of Hiv-1 Restriction Factor Samhd1 Generate a Long-Lived Activated State. Proc Natl Acad Sci U S A 2014, 111:E1843-E1851.

31. Goncalves A, Karayel E, Rice Gl, Bennett KL, Crow YJ, Superti-Furga G, Burckstummer T: Samhd1 Is a Nucleic-Acid Binding Protein That Is Mislocalized Due to Aicardi-Goutieres Syndrome-Associated Mutations. Hum Mutat 2012, 33:1116-1122.

32. Beloglazova N, Flick R, Tchigvintsev A, Brown G, Popovic A, Nocek B, Yakunin AF: Nuclease Activity of the Human Samhd1 Protein Implicated in the Aicardi-Goutieres Syndrome and Hiv-1 Restriction. J Biol Chem 2013, 288:8101-8110.

33. de Silva S, Hoy H, Hake TS, Wong HK, Porcu P, Wu L: Promoter Methylation Regulates Samhd1 Gene Expression in Human Cd4+ T Cells. J Biol Chem 2013, 288:9284-9292.

34. Franzolin E, Pontarin G, Rampazzo C, Miazzi C, Ferraro P, Palumbo E, Reichard P, Bianchi V: The Deoxynucleotide Triphosphohydrolase Samhd1 Is a Major Regulator of DNA Precursor Pools in Mammalian Cells. Proc Natl Acad Sci U S A 2013, 110:14272-14277.

35. St Gelais C, de Silva S, Hach JC, White TE, Diaz-Griffero F, Yount JS, Wu L: Identification of Cellular Proteins Interacting with the Retroviral Restriction Factor Samhd1. J Virol 2014, 88:5834-5844.

36. Bergamaschi A, Ayinde D, David A, Le Rouzic E, Morel M, Collin G, Descamps D, Damond F, Brun-Vezinet F, Nisole S, Margottin-Goguet F, Pancino G, Transy C: The Human Immunodeficiency Virus Type 2 Vpx Protein Usurps the Cul4a-Ddb1 Dcaf1 Ubiquitin Ligase to Overcome a Postentry Block in Macrophage Infection. J Virol 2009, 83:4854-4860.

37. Casey L, Wen X, de Noronha CM: The Functions of the Hiv1 Protein Vpr and Its Action through the Dcaf1.Ddb1.Cullin4 Ubiquitin Ligase. Cytokine 2010, 51:1-9.

38. Srivastava S, Swanson SK, Manel N, Florens L, Washburn MP, Skowronski J: Lentiviral Vpx Accessory Factor Targets Vprbp/Dcaf1 Substrate Adaptor for Cullin 4 E3 Ubiquitin Ligase to Enable Macrophage Infection. PLoS Pathog 2008, 4:e1000059.

39. Guo H, Wei W, Wei Z, Liu X, Evans SL, Yang W, Wang H, Guo Y, Zhao K, Zhou JY, Yu XF: Identification of Critical Regions in Human Samhd1 Required for Nuclear Localization and Vpx-Mediated Degradation. PLoS One 2013, 8:e66201.

40. Ahn J, Hao C, Yan J, DeLucia M, Mehrens J, Wang C, Gronenborn AM, Skowronski J: Hiv/Simian Immunodeficiency Virus (Siv) Accessory Virulence Factor Vpx Loads the Host Cell Restriction Factor Samhd1 onto the E3 Ubiquitin Ligase Complex Crl4dcaf1. J Biol Chem 2012, 287:12550-12558.

41. Lahouassa H, Daddacha W, Hofmann H, Ayinde D, Logue EC, Dragin L, Bloch N, Maudet C, Bertrand M, Gramberg T, Pancino G, Priet S, Canard B, Laguette N, Benkirane M, Transy C, Landau NR, Kim B, Margottin-Goguet F: Samhd1 restricts the replication of Human Immunodeficiency Virus Type 1 by depleting the intracellular pool of Deoxynucleoside Triphosphates. Nat Immunol 2012, 13:223-228.

42. Kim B, Nguyen LA, Daddacha W, Hollenbaugh JA: Tight Interplay among Samhd1 Protein Level, Cellular Dntp Levels, and Hiv-1 Proviral DNA Synthesis Kinetics in Human Primary Monocyte-Derived Macrophages. J Biol Chem 2012, 287:21570-21574.

43. Laguette N, Sobhian B, Casartelli N, Ringeard M, Chable-Bessia C, Segeral E, Yatim A, Emiliani S, Schwartz O, Benkirane M: Samhd1 Is the Dendritic- and Myeloid-Cell-Specific Hiv-1 Restriction Factor Counteracted by Vpx. Nature 2011, 474:654-657.

44. Diamond TL, Roshal M, Jamburuthugoda VK, Reynolds HM, Merriam AR, Lee KY, Balakrishnan M, Bambara RA, Planelles V, Dewhurst S, Kim B: Macrophage Tropism of Hiv-1 Depends on Efficient Cellular Dntp Utilization by Reverse Transcriptase. J Biol Chem 2004, 279:51545-51553.

45. Kennedy EM, Gavegnano C, Nguyen L, Slater R, Lucas A, Fromentin E, Schinazi RF, Kim B: Rntps as Substrate of Human Immunodeficiency Virus Type 1 Reverse Transcriptase in Human Macrophages. J Biol Chem 2010, 285:39380-39391. 
46. Heinemann V, Xu YZ, Chubb S, Sen A, Hertel LW, Grindey GB, Plunkett W: Inhibition of Ribonucleotide Reduction in Ccrf-Cem Cells by 2',2'-Difluorodeoxycytidine. Mol Pharmacol 1990, 38:567-572.

47. Hofer A, Crona M, Logan DT, Sjoberg BM: DNA Building Blocks: Keeping Control of Manufacture. Crit Rev Biochem Mol Biol 2012, 47:50-63.

48. Rampazzo C, Miazzi C, Franzolin E, Pontarin G, Ferraro P, Frangini M, Reichard P, Bianchi V: Regulation by Degradation, a Cellular Defense against Deoxyribonucleotide Pool Imbalances. Mutat Res 2010, 703:2-10.

49. Van Cor-Hosmer SK, Kim DH, Daly MB, Daddacha W, Kim B: Restricted 5'-End Gap Repair of Hiv-1 Integration Due to Limited Cellular Dntp Concentrations in Human Primary Macrophages. J Biol Chem 2013, 288:33253-33262.

50. Chugh P, Bradel-Tretheway B, Monteiro-Filho CM, Planelles V, Maggirwar SB, Dewhurst S, Kim B: Akt Inhibitors as an Hiv-1 Infected Macrophage-Specific Anti-Viral Therapy. Retrovirology 2008, 5:11.

51. Fromentin E, Gavegnano C, Obikhod A, Schinazi RF: Simultaneous Quantification of Intracellular Natural and Antiretroviral Nucleosides and Nucleotides by Liquid Chromatography-Tandem Mass Spectrometry. Anal Chem 2010, 82:1982-1989.

doi:10.1186/s12977-014-0063-2

Cite this article as: Hollenbaugh et al:: dNTP pool modulation dynamics by SAMHD1 protein in monocyte-derived macrophages. Retrovirology 2014 11:63.

\section{Submit your next manuscript to BioMed Central and take full advantage of:}

- Convenient online submission

- Thorough peer review

- No space constraints or color figure charges

- Immediate publication on acceptance

- Inclusion in PubMed, CAS, Scopus and Google Scholar

- Research which is freely available for redistribution

Submit your manuscript at www.biomedcentral.com/submit
() Biomed Central 